\title{
Avatar - a Multi-sensory System for Real Time Body Position Monitoring
}

\author{
E. Jovanov ${ }^{1}$, N. Hanish ${ }^{2}$, V. Courson ${ }^{2}$, J. Stidham ${ }^{1}$, H. Stinson ${ }^{1}$, C. Webb ${ }^{1}$, K. Denny ${ }^{1}$ \\ ${ }^{1}$ University of Alabama in Huntsville, Huntsville, Alabama, U.S.A. \\ ${ }^{2}$ Northrop Grumman Information Systems, Huntsville, Alabama, U.S.A.
}

\begin{abstract}
Virtual reality and computer assisted physical rehabilitation applications require an unobtrusive and inexpensive real time monitoring systems. Existing systems are usually complex and expensive and based on infrared monitoring. In this paper we propose Avatar, a hybrid system consisting of off-the-shelf components and sensors. Absolute positioning of a few reference points is determined using infrared diode on subject's body and a set of $\mathrm{Wii}^{\odot}$ Remotes as optical sensors. Individual body segments are monitored by intelligent inertial sensor nodes iSense. A network of inertial nodes is controlled by a master node that serves as a gateway for communication with a capture device. Each sensor features a 3D accelerometer and a 2 axis gyroscope. Avatar system is used for control of avatars in Virtual Reality applications, but could be used in a variety of augmented reality, gaming, and computer assisted physical rehabilitation applications.
\end{abstract}

Keywords - Wearable monitoring, Wireless sensors, Body Area Networks, Computer Assisted Rehabilitation, Body monitoring.

\section{INTRODUCTION}

Real-time control of avatars in virtual reality applications requires unobtrusive wearable monitors that can be seamlessly integrated into the system. Some existing solutions include wearable suites by Animazoo ${ }^{\odot}$ [1], IDEEA by MiniSun ${ }^{\odot}$ [2], as well as optical sensor solutions used by many movie studios similar to what Raskar explains here[5]. All three provide great solutions using wearable groups of inertial or optical sensors for motion capturing. The solution from MiniSun uses 5 sets of sensors to calculate body and limb positions. Although the IDEEA from MiniSun is a wearable portable device, it is not real time. Optical solutions are also very good, as evidenced by increased use in the new generation of animated movies. These systems also have serious drawbacks. They are extremely expensive, starting at $\$ 50,000$, and confined to the room where the optical cameras or sensors are permanently mounted. If cameras are used, they require a lengthy setup time, and also the person wearing the suite must undergo a drawn out setup time usually requiring hundreds of markers being placed in various positions around the body. An alternative to optical sensing is to use inertial sensors. Animazoo provides great solutions using groups of inertial sensors but they too are tailored toward high-end businesses. There are still several problems with inertial sensors that must be overcome. The most serious deficiency of inertial systems is lack of absolute position. Vlasic et al. [3] use ultra sonic sensors to determine relative location of sensors on the body. Signal processing is also required as the inertial sensors and the environment do introduce noise into the system as well as motion artifacts. It has been demonstrated that the Kalman filter provides optimal body position and state estimation of inertial sensors [3].

We propose an original approach using hybrid system that employs body area network of inertial sensors and optical sensing of the absolute position using off-the-shelf Wii sensors. This paper presents the concept and prototype system for real time unobtrusive body position monitoring.

\section{METHODS}

Avatar system integrates optical, wired and wireless sensors to achieve a cost-effective, unobtrusive, real-time body position monitoring system (Fig. 1). Absolute positioning of a few reference points is determined using infrared diodes on subject's body while the position and orientation of other body segments is determined using a network of inertial sensors. Gateway node controls a network of inertial sensors and communicates wirelessly via Bluetooth with the capture device, in our system it is a standard laptop with Bluetooth connectivity. Our prototype system monitors only one arm. We use two infrared diodes as markers on the shoulder, and four inertial sensor nodes. Sensor nodes are located on the shoulder, upper arm, lower arm, and hand.

We use two $\mathrm{Wii}^{\odot}$ Remotes as optical sensors to determine absolute position of the shoulder reference points. We use two IR diodes to maintain visibility of at least one diode at all times. The remotes have known fixed position. Reference point position is determined by triangulation of two-dimensional point outputs from both sensors. Wii ${ }^{\circ}$ Remotes communicate with the capture device wirelessly using Bluetooth interface.

Inertial sensors - iSense are designed as intelligent 5 degree of freedom (5DOF) sensors (Fig. 2 and Fig. 3). iSense features a low power microcontroller MSP430F2274 for signal conditioning, processing and communication. A network of iSense modules communicate with the gateway through the shared I2C bus (Fig. 4). 


\section{Avatar - Real-Time Motion Capture System

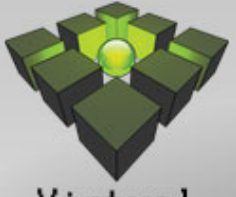

Virtual

Reality

Interface

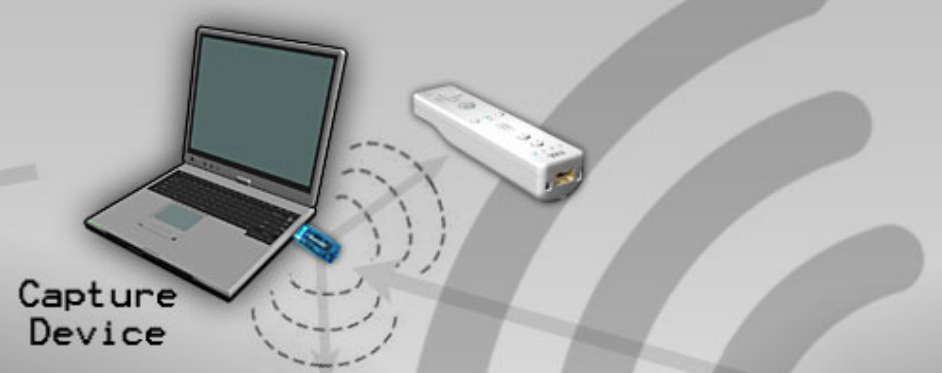

Gat eway Node
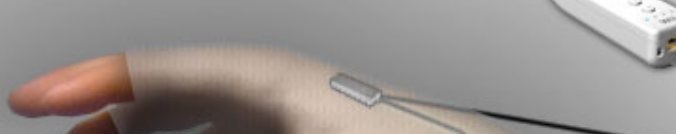

.

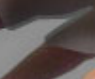

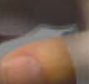

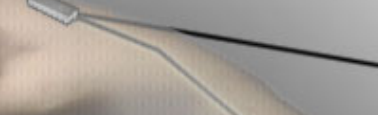

\section{UAHuntsville}

Fig. 1. Block diagram of the hybrid monitoring system.

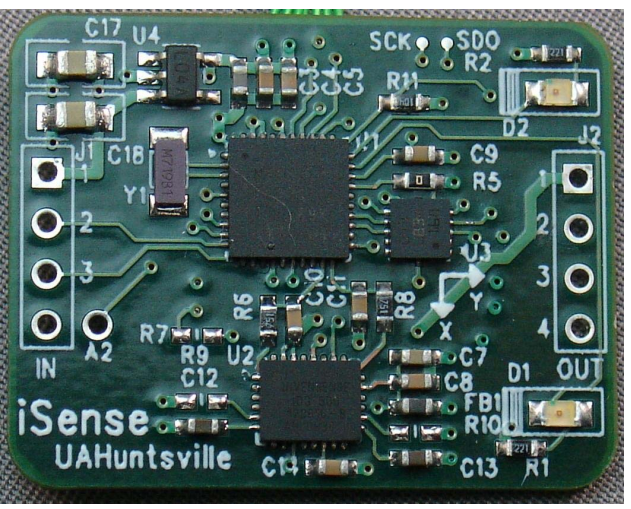

Fig. 2. iSense - inertial sensor module.

Each module contains a 3D accelerometer (Bosch SMB380) and a two axis gyroscope (InvenSense IDG500). Looking at our final board, each sensor weighs less than an ounce and is $1.20 \times 0.94$ inch in size.

The gateway controls a network of iSense nodes as I2C master, processes data from individual sensors and communicates with the data capture device through Bluetooth wireless interface. The gateway node features an ARM7 processor NXP LPC2368 with 58 KB RAM and 512 $\mathrm{KB}$ program memory. The processor runs at $72 \mathrm{MHz}$ that allows execution of complex algorithms in real-time, while power efficient modes extend battery life.

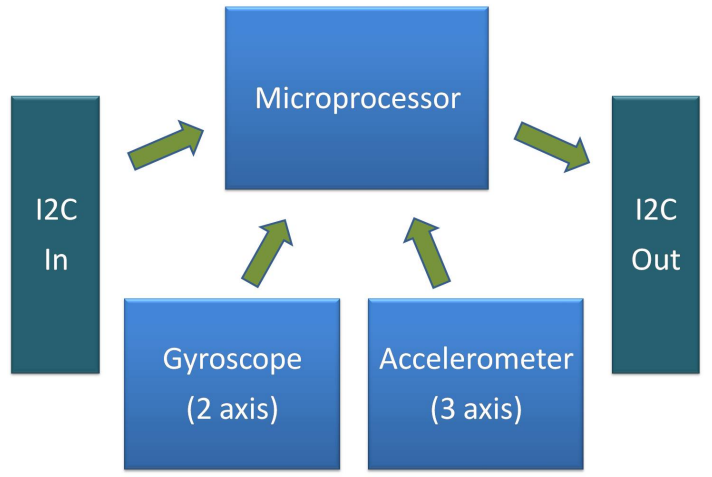

Fig. 3. Block diagram of the iSense inertial sensor

We estimate sensor orientation using relative angles of the gravity vector, $\alpha$ and $\theta$, as represented in Fig. 5. Angles and angular velocities are represented as states of the Kalman filter, as presented in (1).

$$
X_{k}=\left[\begin{array}{c}
\alpha_{k} \\
\dot{\alpha}_{k} \\
\theta_{k} \\
\dot{\theta}_{k}
\end{array}\right]
$$




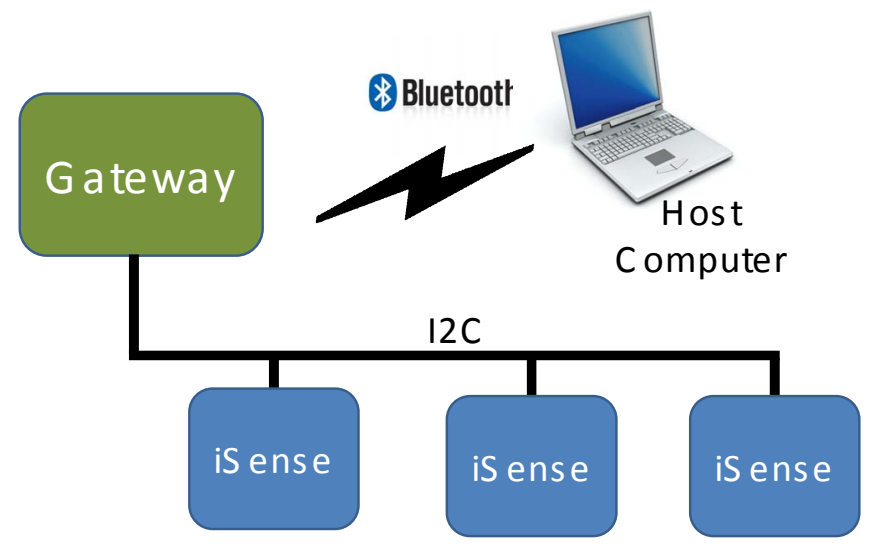

Fig. 4. Avatar system communication.

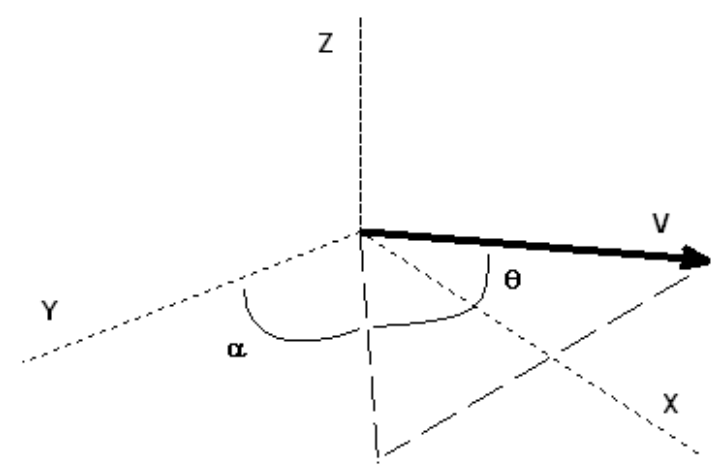

Relative angle is estimated using two accelerometer components (e.g. $\mathrm{X}$ and $\mathrm{Y}$ acceleration components for angle $\alpha$ ). We use gyroscope output to estimate angular rate change. This approach significantly improves position estimate during movement, as represented in Fig. 6. Noisy signal (blue line) represents angle estimate from accelerometer components. Direct angle estimate requires inverse tangent function that amplifies noise from accelerometer signals. Kalman estimate of the angle (red line) improves the estimate and de-noises the signal, as described above.

All signals are sampled at $200 \mathrm{~Hz}$. Output is generated every $5 \mathrm{~ms}$ for raw signals and $20 \mathrm{~ms}$ for processed data using Kalman filter. The system created a processing latency of less than $50 \mathrm{~ms}$, which is ideal in motion capture systems. We generate output at lower frequency during run time to extend battery life of the system.

Calibration of sensors became necessary as we discovered that each inertial sensor's axis had different offset and sensitivity. Calibration of the iSense board was done by rotating it at a constant known speed to obtain each axis's maximum and minimum values for $+1 \mathrm{~g}$ and $-1 \mathrm{~g}$. This calibration device was created using a servo motor and controller. Special attention was needed to keep this device in the same plane of rotation.

Fig. 5. Estimation of iSense orientation; $\alpha, \theta$ are relative angles of gravity vector and determine sensor orientation.

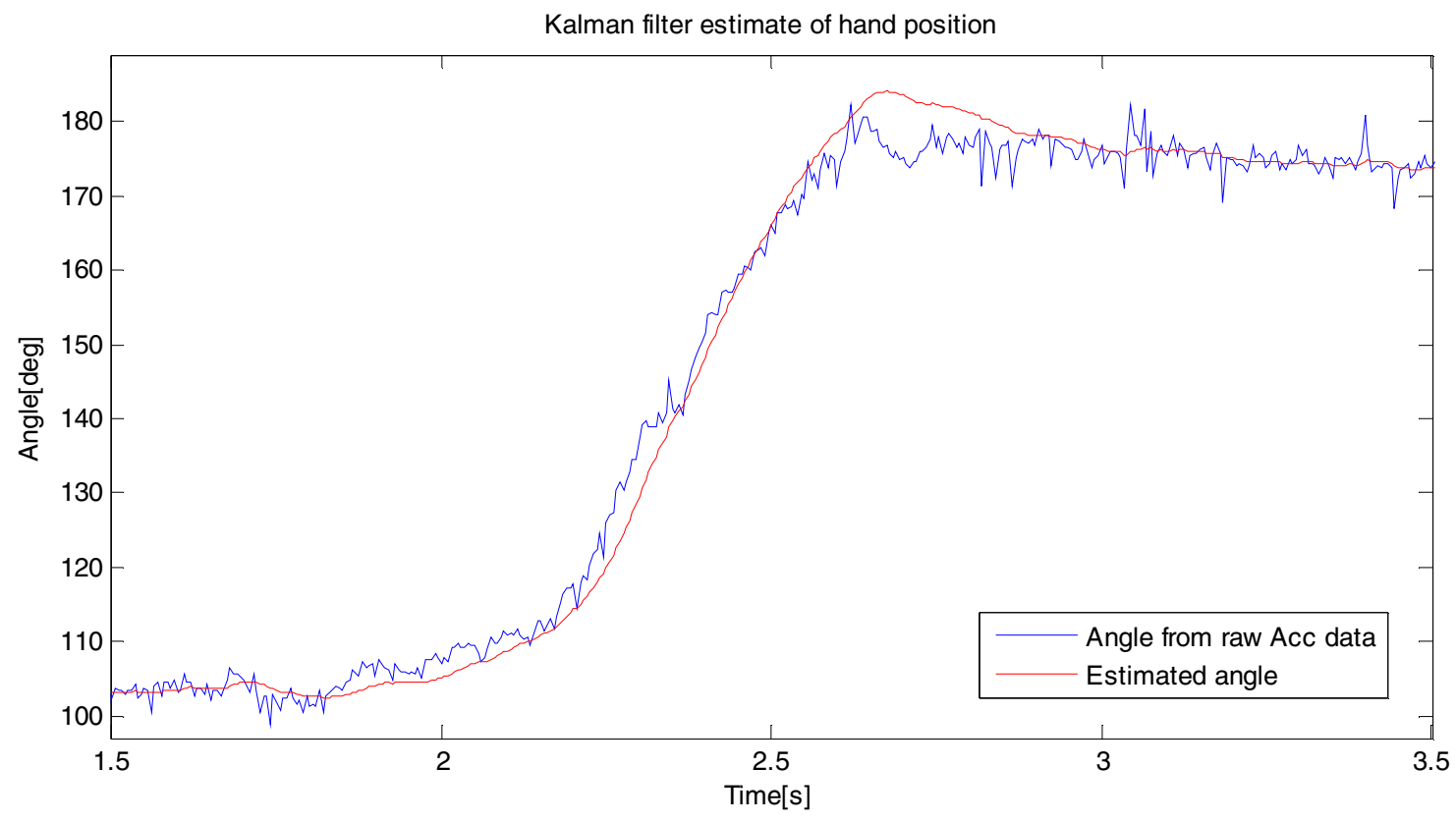

Fig. 6. Kalman estimate of the relative angle and estimate from accelerometer signals 
Testing procedures included attaching the device to outer side of the upper arm and recording the basic motion starting with the arm at rest next to the body, as presented in Fig. 7. Avatar generated on the host computer is also presented on the right side of Fig. 7. We then brought the arm forward, then back to the resting position. The arm was then lifted to the side of the body and returned back to the resting position. During these tests we also captured video so that we could sync recorded signals with the user's sequence of motions. Other tests also included attaching the sensor to the forearm near the elbow and performing a series of wrist rotations.

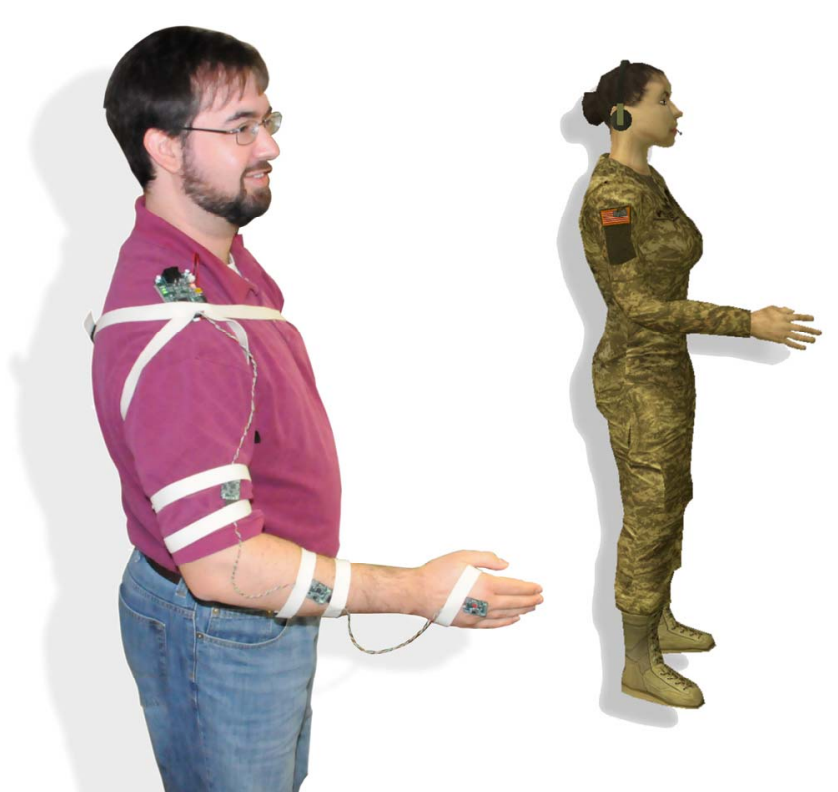

Fig. 7. Prototype system mounted on the right arm (left) and avatar controlled by the system (right).

\section{RESULTS}

Some of our experimental results include testing human rotation and movement capacities. Using an athletic person, we were able to achieve a max wrist rotation of $539 \%$ s. This max rotation will currently saturate our gyroscope, as well as all commercially available devices.

We evaluated average error of the iSense inertial sensors using simplified setup. The average error for the initial set of measurements of the orientation angles ( $\alpha$ and $\theta$ in Fig. 5) was 2.35 degrees with standard deviation of 1.99 degrees. The precision was sufficient for the prototype development; however, it will be necessary to calibrate accelerometers of individual sensors to improve precision of the final system.
Wireless range of Bluetooth module is up to 30 meters, but our range is limited by the type of IR LED we use in conjunction with the Wii remotes. Current experiments have shown that 5 meters is the maximum range of the Wii remote in our system.

We concluded that the Kalman estimator best suited our real-time signal processing needs. All other signal processing methods resulted in unacceptable distortion and/or latency.

\section{CONCULSIONS}

We presented an original approach to real-time body position monitoring. In addition to control of avatars in virtual reality systems, the proposed approach is very convenient for unobtrusive, real-time monitoring for augmented and virtual reality applications (e.g. virtual reality pain management), computer assisted physical rehabilitation, such as post-surgery and stroke rehabilitation, as well as wellness management.

We plan to expand our system to full body monitoring and support simultaneous monitoring of multiple subjects.

\section{ACKNOWLEDGMENT}

We are grateful to Matthew Fredrickson for master server code and Future Design Inc, Huntsville, AL for iSense development and system integration.

\section{REFERENCES}

[1] Animazoo, Available: http://www.animazoo.com, (April 2009)

[2] MiniSun, Available: http://www.minisun.com, (April 2009)

[3] D. Vlasic, R. Adelsberger, G. Vannucci, J. Barnwell, M. Gross, W. Matusik, J. Popović, "Practical motion capture in everyday surroundings," Proc. of the 2007 ACM SIGGRAPH Conference, Article No. 35.

[4] R.Y. Slyper, J.K. Hodgins, "Action Capture with Accelerometers," Proc. 2008 ACM SIGGRAPH / Eurographics Symposium on Computer Animation, July 2008.

[5] R. Raskar, H. Nii, B. Dedecker, Y. Hashimoto, J. Summet, D. Moore, Y. Zhao, J. Westhues, P. Dietz, J. Barnwell, S. Nayar, M. Inami, P. Bekaert, M. Noland, V. Branzoi, E. Bruns, "Prakash: lighting aware motion capture using photosensing markers and multiplexed illuminators," ACM Transactions on Graphics, 26 (3), 2007, Art. 36.

[6] K. Hoshino, "Dexterous Robot Hand Control with Data Glove by Human Imitation," IEICE - Transactions on Information and Systems, Volume E89-D, Issue 6, June 2006, pp. 1820-1825. 